\title{
Caracterización morfométrica de estructuras florales de Tagetes erecta L. y Tagetes patula L. (Asteraceae) utilizando análisis digital de imágenes y dimensión fractal
}

\section{Morphometric characterization of floral structures Tagetes erecta L. and Tagetes patula L. (Asteraceae) using digital image analysis and fractal dimension}

\author{
Maria Ximena Quintanilla Carvajal ${ }^{1,2}$, Martha lucía Arenas Ocampo ${ }^{2}$, Roberto Campos Mendiola ${ }^{2}$, \\ Brenda Hildeliza Camacho Díaz ${ }^{2}$, Antonio Ruperto Jiménez Aparicio*
}

${ }^{1}$ Facultad de Ingeniería, Universidad de La Sabana, Campus del Puente del Común, km. 7, Autopista Norte de Bogotá. Chía, Cundinamarca, Colombia.

${ }^{2}$ Centro de Desarrollo de Productos Bióticos. Ceprobi 8, Col. San Isidro, Yautepec, Morelos, México.

*aaparici@ipn.mx

\begin{abstract}
RESUMEN
Las flores Tagetes erecta L. y Tagetes patula L. tienen usos ornamentales en México, y también algunas industrias las utilizan en la coloración de carne. El género Tagetes presenta especies y variedades con diferentes colores y tonalidades en sus flores. Por lo que, una forma de distinción rápida y objetiva de estas variedades sería útil para la industria. En este trabajo se desarrolló un método para distinguir y caracterizar la diversidad en dos variedades de T. erecta y T. patula a través de la caracterización de las estructuras florales, utilizando técnicas de análisis digital de imágenes y análisis fractal. Para esto, se capturaron imágenes de lígulas y capítulos florales, que posteriormente fueron binarizadas para obtener imágenes de objetos separados (lígulas y capítulos florales). El análisis de estas imágenes se realizó determinando diferentes parámetros morfométricos, tales como perímetro, área proyectada, longitud máxima, factor de forma y compacidad. Adicionalmente, se determinó la dimensión fractal de contorno, de perímetro y de área. Los resultados mostraron que la dimensión fractal y el factor de forma son los parámetros que detectan con mayor sensibilidad las diferencias entre lígulas y entre capítulos florales, por lo que se proponen como parámetro adecuados para caracterizar estructuras florales.
\end{abstract}

Palabras clave: Tagetes erecta, T. patula, estructuras florales, análisis digital de imágenes, análisis fractal.

\begin{abstract}
Tagetes erecta L. and Tagetes patula L. flowers have ornamental uses in Mexico, but also some industries used in the coloring of meat. The genus Tagetes presents species and varieties with different colors and shades in their flowers. Therefore a rapid and objective form of distinction of these varieties would be useful for the industry. In this research was developed a method to distinguish and characterize the diversity in two varieties of the T. erecta and T. patula through the characterization of the floral structures, using techniques of digital image analysis and fractal analysis. Images of ligules and flower heads were captured and binarized to produce images of separate objects (ligules and flower heads). The analysis of these images was performed by determining different morphometric parameters such as perimeter, projected area, maximum length, shape factor and compactness. Additionally, the boundary fractal dimension, perimeter fractal dimension and area fractal dimension were determined. The results showed that the fractal dimension and form factor were the parameters in petals and flower head with higher sensitivity to detect differences between and among varieties and so are proposed as suitable parameter to characterize floral structures.
\end{abstract}

KEYWORDs: Tagetes erecta, T. patula, floral structures, digital image analysis, fractal analysis.

\section{INTRODUCCIÓN}

El género Tagetes (Asteraceae) se compone de aproximadamente 30 especies, entre las cuales, cerca de la mitad está en México (Turner 1996). Las especies más conocidas son la T. erecta L., T. patula L., T. lunulata Ortega y T. tenuifolia Cav., siendo la primera la más utilizada (Serrato-Cruz \& Miranda-Colin 1998). Tagetes erecta, también conocida como (cempasúchil) "flor del muerto", se caracteriza por su coloración y su contenido de carotenoides (Delgado et al. 2000). Esta flor se utiliza en la pigmentación de la carne del pollo, tilapia y otras clases de pescados; también se ha utilizado para pigmentar otros productos como la yema de huevo (Ponce et al. 2004). Dicha pigmentación se debe a sus carotenoides que presentan actividad antioxidante (Delgado \& Paredes 1996, Delgado et al. 2000); de provitamina "A" (Serrato-Cruz \& Barajas-Pérez 2006). Las variedades cultivadas de esta planta en México se han 
originado por el cruzamiento de poblaciones naturales y cultivadas; esto ocasiona dificultad para distinguir entre sus variedades (Serrato 1998). Una característica de distinción entre dichas variedades es la forma de los capítulos florales y de las lígulas que componen cada especie. Las flores de Tagetes erecta son inflorescencias de tonos naranjas, amarillos y rojos. Las inflorescencias tienen capítulos con numerosas flores individuales (de 60 a 400). Estas flores pueden o no tener lígulas o pétalos (pétalas o apétalas). En el caso de $T$. patula, los capítulos tienen lígulas con el limbo elíptico de 8 a18 $\mathrm{mm}$ de largo y 6 a10 $\mathrm{mm}$ de ancho; las flores con forma de disco son de 12 a $40 \mathrm{~mm}$, y las tubulares de 10 a15 mm de largo (Gliman \& Howe 1999, Villareal \& Villaseñor 2004).

Normalmente, la identificación botánica de cualquier planta se apoya en una descripción morfológica de algunos órganos, como las flores. En años recientes se ha utilizado el análisis digital de imágenes (ADI) para describir el tamaño y la forma de objetos y materiales biológicos (Alvarado \& Aguilera 2001, Davies 2000, Pérez-Nieto et al. 2010, Mery et al. 2010, Camacho-Díaz et al. 2010, Quintanilla-Carvajal et al. 2011). Con el ADI se pueden obtener parámetros morfométricos de tamaño y forma. El ADI se puede complementar con el análisis fractal que es útil para describir objetos con formas complejas (Panigrahi et al. 1998, JiménezMartínez et al. 2009, Pérez-Nieto et al. 2010, QuintanillaCarvajal et al. 2011).

Algunos autores como Núñez-Colin et al. (2011), realizaron mediciones en partes florales usando diámetros, perímetros, Feret, índice de alargamiento con eje mayor y menor, índice de redondez, índice de compactación. Estos tres últimos son herramientas para caracterizar la forma de objetos.

El objetivo de este trabajo fue determinar características morfométricas de algunas variedades de flores de Tagetes erecta y T. patula utilizando técnicas de ADI y análisis fractal con el fin de proponer el uso de estas técnicas como nuevas herramientas en la identificación de variedades de plantas con flores.

\section{MATERIALES Y MÉTODOS}

\section{Materiales}

Se utilizaron como materiales biológicos: a) inflorescencias de Tagetes erecta de color blanco, amarillo y naranja con capítulos florales dobles, simples y apétalos; y con lígulas lisas y convolucionadas; b) inflorescencias de Tagetes patula de color amarillo y rojo con lígulas convolucionadas y lisas (Serrato et al. 2006). Para cada tipo se tomaron 22 capítulos florales. En el caso de las lígulas, se muestreó un 10\% de los capítulos florales, tomando 40 lígulas al azar estimando que el error típico estuviera alrededor de $8 \%$ (Alvarado \& Aguilera 2001). Este material se obtuvo de una recolección nacional cuyo germoplasma se cultivó durante los meses de junio a noviembre de 2006 en los campos experimentales de la Universidad Autónoma de Chapingo, Estado de México. El muestreo, se realizó completamente al azar y tomando en consideración la división propuesta por Serrato et al. (2006). CAPTURA Y PROCESAMIENTO DE IMÁGENES

Los capítulos florales completos se separaron de los tallos y se pusieron sobre un fondo negro mate. Las imágenes se capturaron bajo luz diurna indirecta usando una cámara reflex digital, marca Nikon modelo D50 con una lente Nikon, modelo AF-S de 18-55 mm/f3.5-5.6 (Nikon. Japón). La distancia focal se mantuvo constante y perpendicular entre la cámara y los capítulos florales. Para cada imagen capturada se colocó una regla (escala en $\mathrm{mm}$ ) para poder calibrar el análisis digital de imágenes. Las imágenes se guardaron en formato jpg y tamaño de imagen de 1260 x 980 píxeles, en color 32 bits.

En el caso de las lígulas, se separaron del capítulo floral y se pegaron con adhesivo en hojas de fondo negro para tener un buen contraste. Posteriormente las plantillas que contenían a las lígulas se llevaron al escáner digital y las imágenes se capturaron en exposición automática y tamaño de 1260 x 980 píxeles, en formato jpg, color 24 bits. También se colocó un referente de escala para la posterior calibración en el ADI.

Para realizar el ADI, las imágenes de capítulos florales o lígulas primero fueron procesadas como objetos. Cada objeto fue separado del resto de la imagen usando la herramienta borrador del programa Corel Draw. V11.5 (Corel Draw Corporation USA). Las imágenes de cada objeto se pegaron en una nueva carpeta, y se guardaron en formato jpg de color de 24 bits. Posteriormente las imágenes se binarizaron utilizando la herramienta "rellenar" y se guardaron en formato *.bmp, escala de grises de 8 bits, para después realizar el ADI.

ANÁLisis DigitAl DE IMÁGENES

El ADI consistió en la determinación de las dimensiones y de las formas de los objetos (capítulos florales y las lígulas) y para esto se utilizó el programa Sigma Scan Pro V5.0 (SPSS, USA). Para la determinación de las dimensiones se midió: 1) perímetro máximo (Pmáx), que es la distancia formada por los puntos que delimitan el contorno de un objeto; 2) área (A), que son los puntos contenidos en el objeto; 3) longitud máxima (Lmáx) que es la distancia entre dos puntos más alejados del objeto. Para la determinación de la forma de capítulos florales y lígulas se aplicaron algunos descriptores morfométricos contenidos en el menú "settings"; 4) factor de compacidad o compactación $(\mathrm{Fc})$ que es el cociente del perímetro al cuadrado por el área (P2/A), en donde la forma circular perfecta tiene valor de 12.5, pero en objetos irregulares los valores son mayores; 5) factor de forma (Fs), que es el cociente $4 \pi \mathrm{A} / \mathrm{P} 2$, en donde los objetos con forma circular tienen valores cercanos a 1, mientras los objetos que no son circulares tienden a 0 .

ANÁLISIS FRACTAL DE LAS IMÁGENES.

El análisis fractal consistió en la evaluación de la dimensión fractal de contorno, perímetro y área de las lígulas y de los capítulos florales. 
a) Dimensión fractal de contorno.

Con el programa Image J V1.34 (National Asociation of Health. USA) se procedió a determinar la Dimensión fractal de contorno (Dfc) por el método de conteo de cajas ("boxcounting"). Para ello, a las imágenes binarizadas ( 2 bits) se les separó el contorno, utilizando la herramienta "outline". La Dfc se evaluó con un tamaño de cajas que se iban incrementando en una progresión geométrica de 2 hasta 64 píxeles (Lira 2002). Dicho método se basa en el concepto matemático de cubrir un objeto dentro de una imagen con rejillas de tamaños diferentes, contando cada orificio como una caja. Y que al disminuir el tamaño de caja se necesitan más cajas para cubrir al objeto. Por lo tanto, el método Boxcounting involucra cubrir el objeto por un conjunto de cajas de lado $\varepsilon$; el número de cajas $\mathrm{N}(\mathcal{E})$ es proporcional a la escala

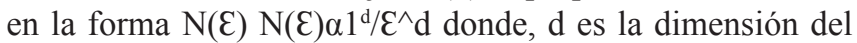
objeto (Gil et al. 2006). Considerando que en el caso de una imagen digital el tamaño de caja corresponde al número de pixeles por pulgada cuadrada.

b) Dimensión fractal de perímetro (Dfp) y de área (Dfa) Se determinó Dfp a través del gráfico del logaritmo natural del área contra el logaritmo natural del perímetro máximo de cada capítulo y lígula; y se aplicó la regresión lineal para obtener la pendiente (m). El valor de Dfp se calculó con $1+$ m. La Dfa se obtuvo al graficar el logaritmo natural del área A) contra el logaritmo natural de la longitud máxima (Lmáx) de cada capítulo y lígula; de igual manera a la regresión lineal obtenida se le determinó la pendiente (m), y la Dfa se obtuvo como 2 - m (Lira 2002).

\section{ANÁLISIS ESTADÍSTICO}

Los valores resultantes, para los capítulos florales y lígulas, fueron analizados con ayuda del programa Excel para análisis estadístico (Promedio, desviación estándar y error típico). Se emplearon pruebas paramétricas (Duncan, t-student) y no paramétricas (Kruskal Wallis) para evaluar diferencias significativas entre las lígulas y entre capítulos con un grado de confiabilidad del 95\% y un error mínimo al 8\%. Dichas pruebas se realizaron con el Programa Sigma Stat SPSS, V.11.5 (Statistical Product and Service Solutions Inc. USA).

Se realizó un dendrograma para lígulas y uno para capítulos florales con los valores de Dfc, Dfp y la Dfa utilizando el programa SPSS-Statistics, V.22. y el método clasificación del vecino más lejano.

\section{RESULTADOS}

LíGULAS

Las imágenes de lígulas capturadas fueron separadas en grupos con características visuales diferentes. La variedad $T$. erecta o T. patula, como grupos separados, se le asignó en el nombre la primera letra E o P, respectivamente. Después, si el pétalo era doble o simple, las letras $\mathrm{D}$ o $\mathrm{S}$, respectivamente.
Y por último, si los pétalos eran lisos o rugosos, adicionando las letras L o R, respectivamente. Se obtuvieron 6 grupos: a) T. erecta, pétalo doble, liso (EDL); b) T. erecta, pétalo simple, liso (ESL); c) T. patula, liso (PL); d) T. erecta, pétalo doble, rugoso (EDR); e) T. patula, rugosa (PR); f) T. patula, apetalada (PA). La morfología típica de los grupos de lígulas se muestra en la Figura 1.

a) Perímetro máximo (Pmáx)

Los resultados para el perímetro máximo de las lígulas se muestran en la Tabla I. De acuerdo con la prueba de Duncan, se pudieron establecer 3 grupos de lígulas. En donde, las lígulas EDL, ESL, PL y PR presentaron valores similares de Pmáx pero significativamente menores a PA. Finalmente EDR presentó el mayor valor de Pmáx. Esto se debe a las prolongaciones y el tamaño de la lígula.

b) Área (A) y Longitud máxima (Lmáx)

El otro descriptor de tamaño fue el área de las lígulas. Los resultados de la prueba de Duncan mostraron que todos los grupos típicos tienen valores de Área significativamente diferentes y sólo hay similitud entre ESL y PL, como se muestra en la Tabla I. Es de resaltar que el parámetro de área es un descriptor más sensible a las diferencias de los distintos tipos de lígulas, mientras que Pmáx tiene menor sensibilidad posiblemente debido a que más de una característica contribuye al resultado del descriptor.

De la misma manera los diversos grupos de lígulas mostraron valores diferentes de Lmáx, por lo que no existen grupos de lígulas con valores Lmáx similares.

c) Factor de compacidad (Fc)

Se evaluó el Fc como un descriptor de la forma de las lígulas ya que con este es posible evidenciar que tan liso o convolucionado es el perímetro. Para objetos lisos sus valores tienden a 12 , mientras que para objetos convolucionados sus valores son más altos debido a que el perímetro es mayor. El análisis de la prueba de Duncan evidenció tres grupos característicos y significativamente diferentes, en donde, ESL, PL y EDL representan un primer grupo con los valores más bajos de $\mathrm{Fc}$, mientras que PR y EDR presentaron los valores de Fc más altos. También se podría pensar que más de una característica contribuye al valor final de este descriptor. Por lo tanto, es también un descriptor de baja sensibilidad para las lígulas.

d) Factor de forma (Fs)

También se evaluó el Fs en las lígulas como otro descriptor de la irregularidad. Si Fs es cercano a 1, la forma del objeto sería esférica u ovalada, mientras que los valores menores de 1 y tendientes a cero se deben a formas diferentes a las ovaladas. En el caso de las lígulas, se esperaría que fueran tendientes a cero por tener una forma alargada.

El análisis de la prueba de Duncan presentó diferentes valores de Fs para cada grupo de lígulas (Tabla I). En donde, 


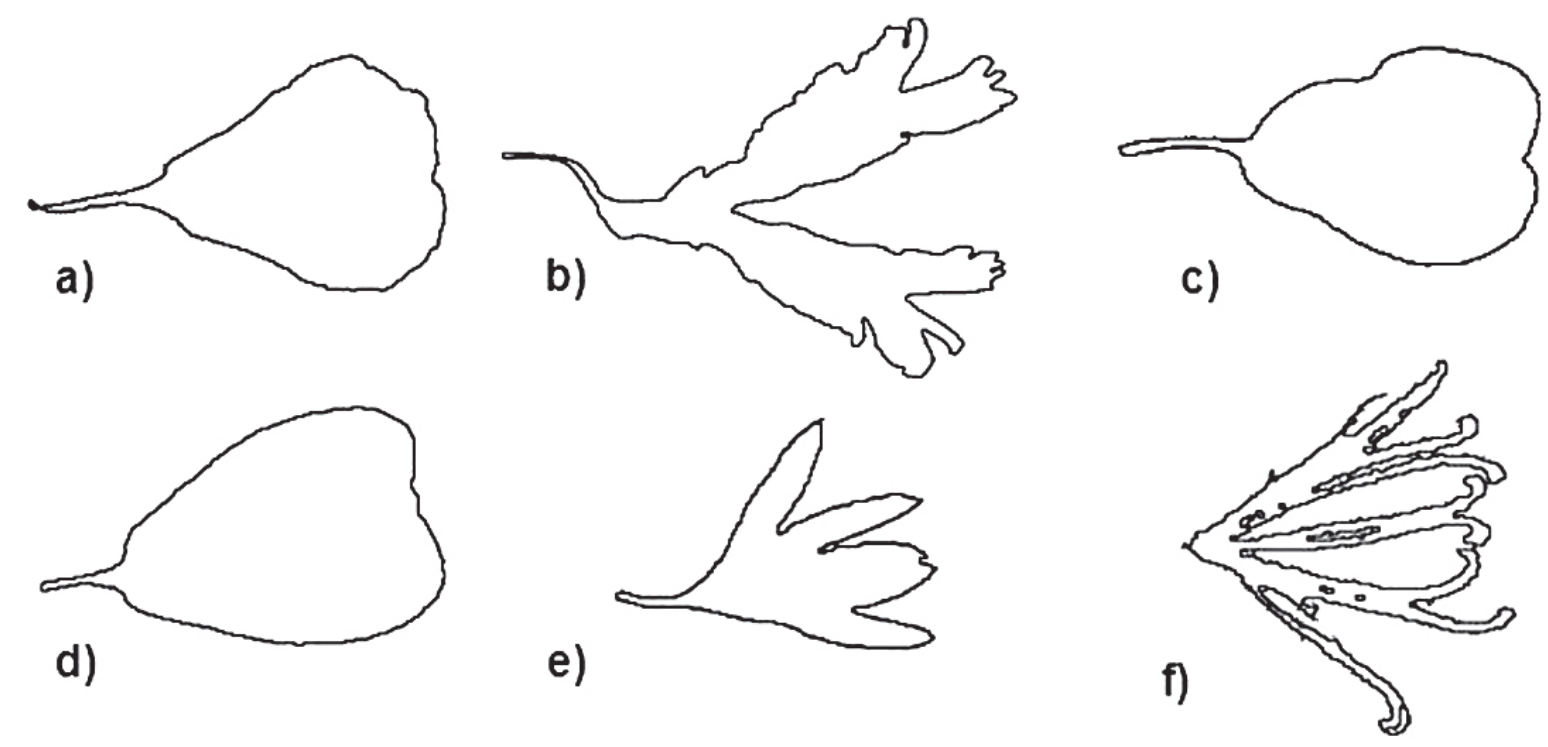

Figura 1. Imágenes que muestran la morfología de las lígulas de Tagetes: a) EDL; b) EDR; c) ESL; d) PL; e) PR y F) PA.

Figure 1. Images that showed the Tagetes ligules morphology: a) EDL; b) EDR; c) ESL; d) PL; e) PR y F) PA.

TABLA I. Comparación de parámetros morfométricos en lígulas para diferenciar variedades de T. erecta y T. patula mediante la prueba de Duncan.

TABLE I. Comparation of morphometric parameters on ligules to find differences between T. erecta and T. patula varieties by Duncan test.

\begin{tabular}{lccccc} 
Grupo & $\mathrm{N}$ & \multicolumn{2}{c}{ VARIABLE } & \multicolumn{1}{c}{ Fs } \\
\hline EDL & 40 & $103,99 \pm 8,32^{\mathrm{a}}$ & $214,13 \pm 19,27^{\mathrm{c}}$ & $2,05 \pm 0,1845^{\mathrm{d}}$ & $0,36 \pm 0,0288^{\mathrm{d}}$ \\
ESL & 29 & $111,50 \pm 9,002^{\mathrm{a}}$ & $271,08 \pm 21,69^{\mathrm{d}}$ & $2,65 \pm 0,212^{\mathrm{e}}$ & $0,45 \pm 0,036^{\mathrm{f}}$ \\
PL & 40 & $112,11 \pm 8,74^{\mathrm{a}}$ & $298,06 \pm 23,84^{\mathrm{d}}$ & $2,58 \pm 0,2064^{\mathrm{e}}$ & $0,41 \pm 0,0328^{\mathrm{e}}$ \\
PR & 40 & $120,19 \pm 11,60^{\mathrm{a}}$ & $110,30 \pm 8,82^{\mathrm{b}}$ & $0,99 \pm 0,0693^{\mathrm{b}}$ & $0,16 \pm 0,0128^{\mathrm{c}}$ \\
PA & 40 & $230,83 \pm 19,39^{\mathrm{b}}$ & $53,58 \pm 3,75^{\mathrm{a}}$ & $0,24 \pm 0,0192^{\mathrm{a}}$ & $0,02 \pm 0,0018^{\mathrm{a}}$ \\
EDR & 40 & $251,30 \pm 20,10^{\mathrm{c}}$ & $371,80 \pm 29,74^{\mathrm{e}}$ & $1,43 \pm 0,1144^{\mathrm{c}}$ & $0,10 \pm 0,007^{\mathrm{b}}$
\end{tabular}

a, b,c, d, e: Presentaron diferencias estadísticamente significativas con $\alpha=0,05$ (letras iguales no hay diferencia y letras diferentes, sí la hay, siendo a, el de menor magnitud $\mathrm{y}^{\mathrm{e}}$ el de mayor magnitud. N: corresponde al tamaño de muestra y los subconjuntos se resaltaron en gris (los que no presentaron diferencias significativas por cada parámetro).

AP y EDR tienen tendencia a cero pero también el resto de los grupos tiene diferencias significativas. Fs mostró ser un descriptor sensible para diferenciar las variedades de lígulas.

\section{e) Relación Área / Perímetro (A/Pmáx)}

Otro descriptor que mostró sensibilidad para distinguir los tipos de lígulas fue el cociente de A y Pmáx. Este descriptor proporciona valores de la forma característica de los objetos, independientemente de sus dimensiones y también da una idea de la irregularidad. Si una lígula es regular su valor de A/Pmáx será cercano al valor 1, por el contrario, si la lígula tiene extensiones irregulares el valor de la relación será diferente a uno.

El análisis de la prueba de Duncan presentó diferentes valores de A/Pmáx para cada grupo de lígulas (Tabla I) a excepción de ESL y PL que mostraron valores cercanos entre sí. También se puede ver que EDR y PR son más cercanos a 1 , por lo tanto son más regulares.

Se puede decir que A/Pmáx es un descriptor que refleja las diferencias de forma entre las lígulas.

\section{CAPÍtulos}

Para la evaluación de tamaño y forma de los capítulos florales se sugirió la misma clasificación de las lígulas, exceptuando las flores apetaladas. Las formas típicas de los capítulos se muestran en la Figura 2.

a) Perímetro máximo (Pmáx) y Área (A)

De acuerdo con la prueba de Duncan, los tipos de los capítulos florales no se diferencian con claridad usando los descriptores 


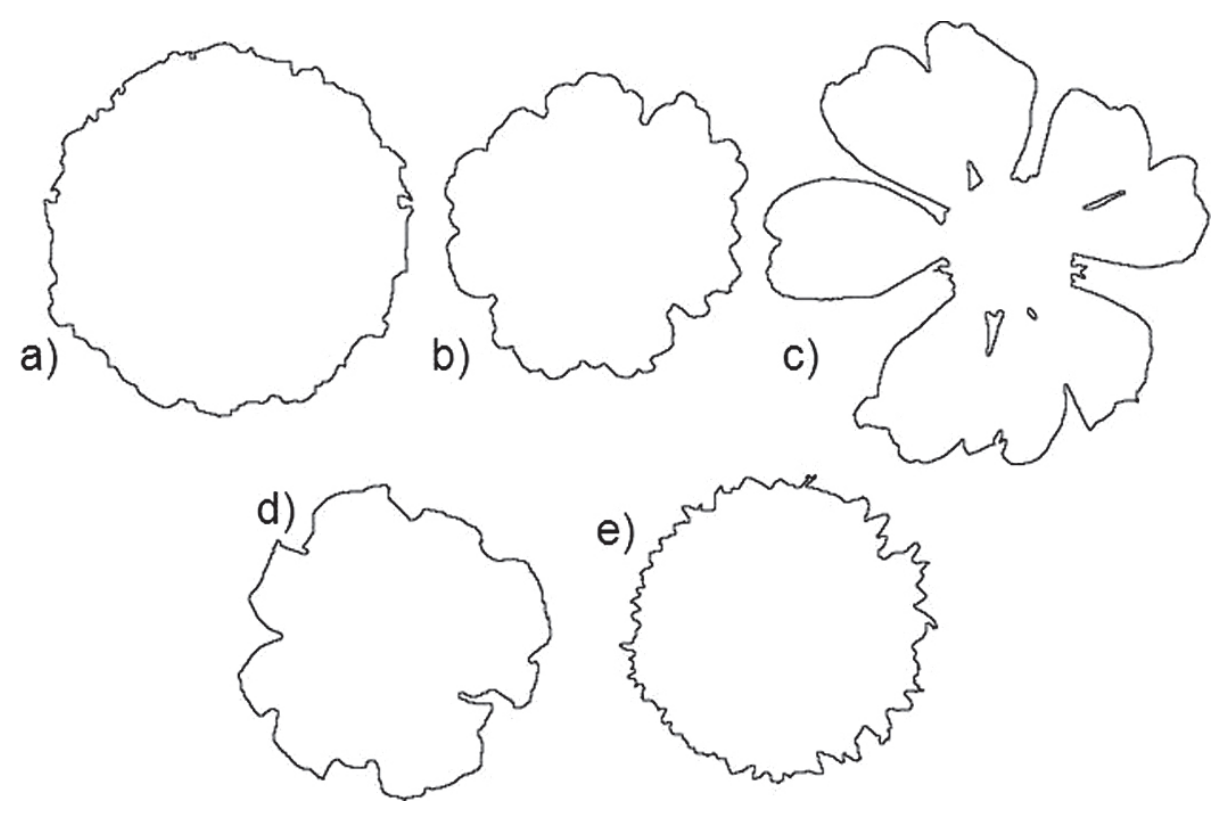

Figura 2. Formas de diferentes capítulos: a) EDL. b) EDC. c) ESL. d) PL. e) PC.

Figure 2. Different forms of flower heads: a) EDL. b) EDC. c) ESL. d) PL. e) PC.

de perímetro máximo (Pmáx) y de Área (A). Por lo tanto, estos descriptores no son sensibles a las características de las variedades de los capítulos florales.

b) Longitud máxima (Lmáx) y Factor de compacidad (Fc) De igual manera que con las lígulas, los parámetros de Longitud máxima (Lmáx) y Factor de compacidad (Fc) demostraron baja sensibilidad para distinguir las características de los capítulos florales.

c) Factor de forma (Fs)

Los resultados para el factor de forma para los capítulos florales no mostraron distinción clara entre los diferentes tipos. Por lo tanto, este descriptor no es sensible a las características de los capítulos florales.

ANÁLISIS DE DIMENSIÓN FRACTAL

Los descriptores de forma Fc y Fs en las lígulas y los capítulos florales proporcionan información de las características de regularidad de la forma, tanto de lígulas como capítulos. Pero hay otros descriptores que pueden proporcionar una mejor caracterización de las formas irregulares. Los descriptores de dimensión fractal que se determinaron fueron de contorno (Dfc), de perímetro (Dfp) y de área (Dfa). Cada uno proporciona información de la irregularidad de un objeto independientemente de sus dimensiones y de su complejidad. Es decir, puede producir datos característicos de objetos que sean muy diferentes al área de un círculo.
TABLA II. Valores de dimensión fractal de contorno para lígulas.

TABLE II. Values of fractal dimension of contour for ligules.

\begin{tabular}{lllll} 
Grupo & CARACterísticas & DFC & Dfp & Dfa \\
\hline EDL & Erecta- Doble- Lisa & 1,1033 & 1,3814 & 1,7839 \\
EDR & Erecta- Doble- Rugoso & 1,2017 & 1,5883 & 1,5452 \\
ESL & Erecta- Simple- Lisa & 1,0889 & 1,4347 & 1,7108 \\
PL & Patula- Lisa & 1,0828 & 1,3052 & 1,7919 \\
PR & Patula- Rugoso & 1,2124 & 1,3932 & 1,5721 \\
PA & Apétala & 1,3912 & 1,2782 & 1,4833
\end{tabular}

a) Lígulas

Los resultados de las diferentes evaluaciones de la dimensión fractal de contorno se consignan en la Tabla II. Se puede ver que los contornos de las lígulas presentan una tendencia de contornos lisos en PL y EDR. De acuerdo con lo observado, se puede formar un primer subconjunto con PL, ESL y EDL que presentaron los valores más pequeños de DFc, es decir, con bordes lisos, y un segundo subconjunto de lígulas más rugosas, formado por EDR y PR, con Dfc de 1,2017 y 1,2124, respectivamente. Las lígulas con más alta irregularidad son del grupo AP. Los valores de Dfc son una buena manera de caracterizar las formas irregulares, como son las lígulas florales (Longley \& Batty 1989).

En la dimensión fractal de la relación área/perímetro máximo (Dfp) en lígulas a diferencia de la Dfc, no se evidenciaron grupos de valores cercanos (Tabla II). Cabe señalar que en este sentido, la Dfp es una relación entre 
propiedades geométricas (área y el perímetro) y proporciona evidencias sobre qué tan liso, rugoso o convolucionado es un perímetro en relación con el área. Es decir, qué tan compacto y qué tan ramificado puede ser (Lira 2002, QuintanillaCarvajal et al. 2011). Los resultados encontrados mostraron que Dfp es sensible a las características de forma, motivo por el cual probablemente cada tipo de lígula presentó un valor específico de Dfp promedio. Por ejemplo, en el caso de PA el valor de Dfa está indicando que se trata de un objeto ramificado; es decir PA llena menos espacio de un área, puesto que su morfología es bastante convolucionada, lo que se explica por el alto valor de Pmáx, por lo tanto estas lígulas son más ramificadas.

b) Capítulos

Al igual que para las lígulas se analizaron los capítulos florales determinando su dimensión fractal de Dfc, Dfp y Dfa; los resultados se muestran en la Tabla III.

TABLA III. Valores de dimensión fractal para capítulos.

TABLE III. Fractal dimension values for flower heads.

\begin{tabular}{lllll} 
GruPo & CARACtERÍsticas & DFC & Dfp & Dfa \\
\hline EDL & Erecta- Doble- Lisa & 1,0930 & 1,1433 & 1,5275 \\
EDR & Erecta- Doble- Rugoso & 1,1727 & 1,2656 & 1,4710 \\
ESL & Erecta- Simple- Lisa & 1,1238 & 1,6464 & 1,4498 \\
PL & Patula- Lisa & 1,1208 & 1,4757 & 1,4642 \\
PR & Patula- Rugoso & 1,1629 & 1,1357 & 1,6080
\end{tabular}

De la misma forma que en el caso de las lígulas, se tuvieron comportamientos acordes con la rugosidad del perímetro (Dfc), el llenado de un plano (Dfp) y la compactación/ramificación (Dfa). Es importante mencionar que por limitaciones técnicas se tuvo una alta variabilidad del tamaño de las muestras (n), por lo que los valores de los coeficientes de correlación $\left(\mathrm{R}^{2}\right)$ de las regresiones mostraron una alta dispersabilidad de los datos.

En el caso de Dfc, los resultados para los capítulos lisos (EDL, ESL y PL) presentaron el menor valor de dimensión fractal, es decir perímetros más lisos que tienden al valor de 1,0; mientras que los capítulos EDR y PR tuvieron valores superiores debido a que su perímetro fue más rugoso y por lo tanto su forma fue más irregular. En el caso de los capítulos florales lisos tienen el perímetro menos rugoso y por consiguiente abarcaron un menor número de cajas (Alvarado \& Aguilera 2001).

Para Dfp, los valores dependían en qué tanto llenan un plano y para Dfa qué tan compacto o ramificado era el capítulo (Fig. 3). Por ejemplo, EDL y PR mostraron el valor más bajo de Dfp debido a que llenan más una forma circular (plano), y por lo tanto tienden a la unidad. De igual manera presentaron los mayores valores de Dfa, por lo que se pueden considerar como objetos compactos y por ende, su valor tiende a ser 2 .

Por el contrario ESL y PL evidentemente, llenan menos dicho círculo (Dfp es mayor), al generarse mayores espacios en el plano, la Dfa es menor y por lo tanto son más ramificados.

Los dendrogramas obtenidos en la Figura 4 para lígulas, mostraron dos grupos principales de las variedades: EDL(1), PL(4) y ESL(3) tuvieron características cercanas, mientras $\operatorname{EDR}(2)$ y PR(5) mostraron cercanía entre sí. Por su parte el dendrograma de la Figura 5 de los capítulos mostró otros dos grupos de variedades con características relacionadas: EDL(1), PR(5) y EDR(2) quienes mostraron cercanía en sus características de forma, mientras ESL(3) y PL(4) tuvieron cercanía entre sí. Los resultados obtenidos mediante los dendrogramas diferenciaron por capítulo floral y por lígula a las variedades lisas de las rugosas, resaltando a la variedad EDL(1), la cual denotó cierta rugosidad en el capítulo floral completo, posiblemente influenciado por presentar doble lígula.

\section{DISCUSIÓN}

Mediante la caracterización morfométrica de las estructuras florales de $T$. erecta y $T$. patula se consiguió establecer una subclasificación tanto visual como estadística para lígulas y capítulos. Para las lígulas los descriptores menos eficientes para la diferenciación entre grupos fueron el Perímetro máximo y el Factor de compacidad. Bajo el primer descriptor se establecieron grupos con valores cercanos: EDL, ESL, PL y PR. Y para el Factor de compacidad los grupos de lígulas lisas (EDL, ESL y PL) presentaron valores cercanos por las características morfológicas consistentes entre sí y otro subconjunto con lígulas rugosas (PR y EDR) también mostraron valores cercanos.

Para los capítulos, aparentemente todos los descriptores evaluados mostraron grupos con valores cercanos, como el Factor de forma que al ser una relación entre el Área y el perímetro evidenció dos subconjuntos; el primero de los más convolucionados (ESL) y un segundo subconjunto de los más homogéneos (EDL, PL y PR).

Algunos autores como Núñez-Colin et al. (2011) han realizado mediciones en partes florales usando: diámetros, perímetros, Feret, índice de alargamiento con eje mayor y menor, índice de redondez, índice de compactación. Estos tres últimos usados como herramientas para caracterizar la forma de objetos. Sin embargo aún no se han reportado valores de dimensión fractal como caracterización de la forma, como dato útil para distinguir entre variedades de flores o como una medida de la complejidad de las formas del objeto mismo.

Se determinó mediante dimensión fractal la naturaleza fractal de lígulas y capítulos florales, ya que son cuerpos irregulares que pueden se caracterizados por su perímetro altamente convolucionado y como superficies no Euclidianas. La descripción de la morfología fractal más completa para $T$. erecta y $T$. patula se logró con Dfc. Se comprobó que al igual que la mayoría de los objetos de la naturaleza, las flores son irregulares y no se comportan como cuerpos perfectos repetitivos, cada variedad y especie tienen características 


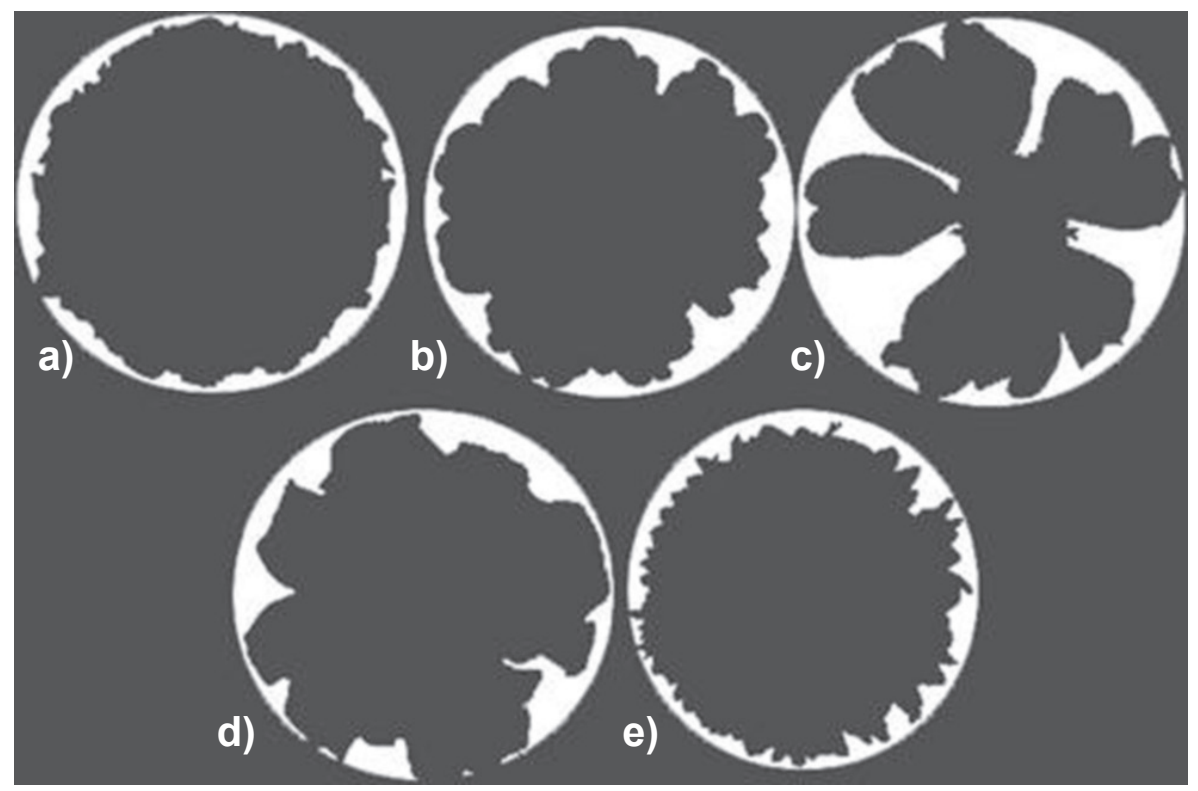

Figura 3. Esquema que muestra el llenado de un plano, la compactación y la ramificación de los diferentes capítulos florales: a) EDL, b) EDR, c) ESL, d) PL y e) PR.

Figure 3. Schematic showing a plane filling, compaction and branching of different flower heads: a) EDL, b) EDR, c) ESL, d) PL e) PR.

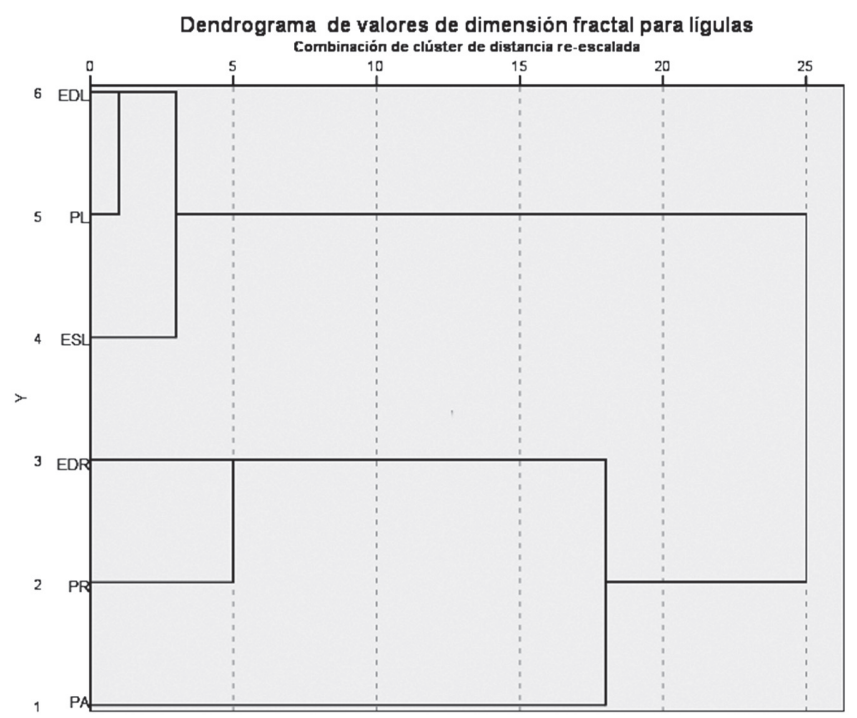

Figura 4. Dendrograma de valores de dimensión fractal para lígulas. FiguRE 4. Dendrogram of fractal dimension values for ligules.

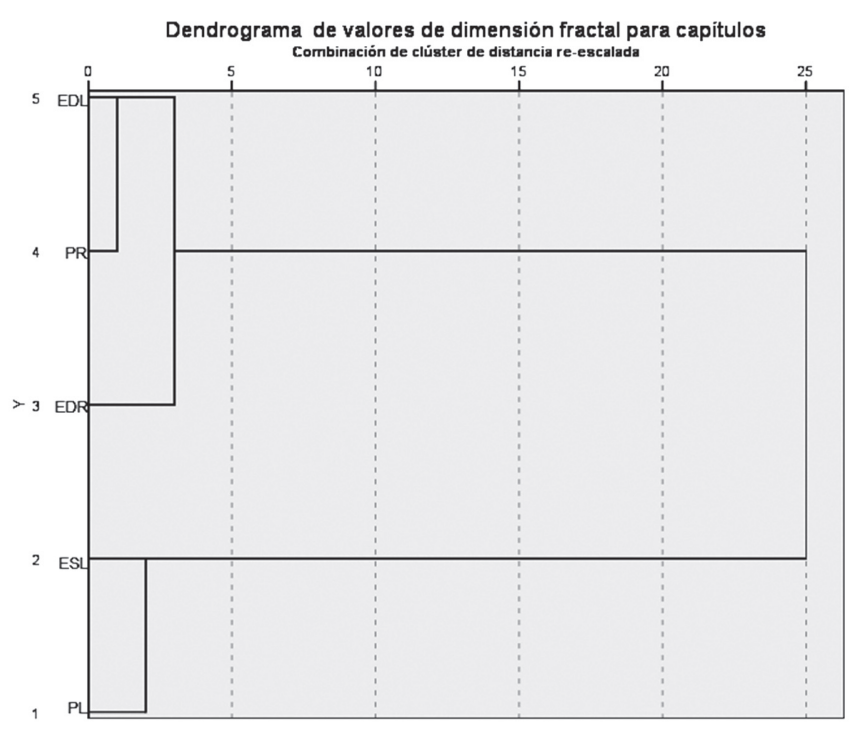

FIGURA 5. Dendrograma de valores de dimensión fractal para capítulo. FIGURE 5. Dendrogram of fractal dimension values for flower heads. 
propias que hacen que cada uno sea singular. Finalmente, el análisis digital de imágenes y el análisis fractal pueden ser herramientas muy versátiles para caracterizar la arquitectura floral de T. erecta y T. patula, por lo que este trabajo propone el uso de la dimensión fractal para complementar el uso de análisis de imágenes como herramienta de estudio para la identificación y caracterización de diversas plantas.

Además los valores de Dfc, Dfp, y Dfa pueden ser usados para relacionar características entre variedades y así ayudar a su clasificación.

\section{BIBLIOGRAFÍA}

Alvarado, J. \& J. Aguilera. 2001. Métodos para medir propiedades físicas en industrias de alimentos. Acribia. España. 410 pp.

Chanona. J., L. Alamilla. \& G. Gutiérrez. 2001. Caos y geometría de fractales, herramientas auxiliares para evaluar la complejidad del entorno biológico. Biotecnología 5(3): 117-126.

Camacho-Díaz, B.H., A. Jiménez-Aparicio, J.J. Chanona-Pérez, G. Calderón-Domínguez, L. Alamilla-Beltrán, H. SÁnchezHernández \& G.F. GutiérRez-López. 2010. Morphological characterization of the growing front of Rhizopus oligosporus in solid media. Journal of Food Engineering 101: 309-317.

DAVIES, E.R. 2000. Image processing for the food industry. World Scientific. Singapore, New Jersey \& Hong Kong. 312 pp.

Delgado-Vargas, F., A.R. Jimenez \& O. Paredes López. 2000. Natural Pigments: Carotenoids, Anthocyanins, and Betalains Characteristics, Biosynthesis, Processing, and Stability. Critical Reviews in Food Science and Nutrition 40(3): 173-289.

Delgado-Vargas F. \& O. Paredes-López. 1996. Correlation of HPLC and AOAC methods to assess the All-trans-lutein content in Marigold flowers. Journal of the Science of Food and Agriculture 72: 283-290.

Gil, J. M. Gimeno, J. Laborda \& J. Nuviala. 2006. Fractal Dimension of Dog Kidney Proximal Convoluted Tubuli Sections by Mean Box-Counting Algorithm. International Journal of Morphology 24: 549-554.

Gliman, E.F. \& T. HowE. 1999. Tagetes erecta. Institute of Food and Agricultural Sciences. Fact Sheet FPS-569: 1-3.

JiméneZ-MartíneZ, C., R. CAampos-Mendiola, M.E. SÁncheZEspíndola, A. Jiménez-Aparicio, G. Gutiérres-López. 2009. Microestructural changes in Lupinus campestris seed in response to three termal debittering treatments. Journal of the Science of Food and Agriculture 89: 2399-2404.

LiRA, J. 2002. Introducción al tratamiento digital de imágenes. Descriptores Morfológicos. Ciencia de la Computación. México. 447 pp.
Longley, P.A. \& M. Batty. 1989. Fractal measurement and line generalization. Computer\&Geosciences. 22 15(2): 167-183.

Merr, D., J.J. Chanona-Pérez, A. Soto, J.M. Aguilera, A. Cipriano, N. Veléz-Rivera, I. Arzate-Vázquez \& G.F. GutiérRez-López. 2010. Quality classification of corn tortillas using computer vision. Journal of the Science of Food and Agriculture 101: 357-364.

NúÑez-Colín, C.A., M.A. Serrato-Cruz, M.T. Santos-Cortés, C.C. Luna-Morales, J. Martínez-Solis, J.A. Cuevas-Sánchez. 2011. Caracterización de Tagetes filifolia del centro-sur de México por morfometría de aquenios. Revista Mexicana de Biodiversidad 82: 539-549.

Panigrahi, S., M. Misra \& S. Willson. 1998. Evaluations of fractal geometry and invariant moments for shape classification of corn germoplasm. Computers and Electronics in Agriculture 20(1): 1-20.

Pérez-Nieto, A., J.J. Chanona-Pérez, R.R. Farrera-Rebollo, G.F. Gutiérrez-López, L. Alamilla-Betrán \& G. CalderónDomínGUEz. 2010. Image analysis of structural changes in dough during baking. LWT-Food Science and Technology 43: 535-543.

Ponce, J.T., J.L. Arredondo \& E.J. Vernon. 2004. Pigmentación de la tilapia (Oreochromis niloticus) con carotenoides de flor de cempaxúchil (Tagetes erecta) en comparación con la astaxantina. Revista Mexicana de Ingeniería Química 3: 219-225.

Quintanilla-Carvajal, M.X., S. Meraz-Torres, L. Alamilla Beltrán, J.J. Chanona-Pérez, E. Terres-Rojas, H. HERNÁNDEZ-SÁNCHEZ, A.R. JimÉNEZ-APARICIO \& G.F. GUTIÉRREZ-LóPEZ. 2011. Morphometric characterization of spray-dried microcapsules before and after $\alpha$-tocopherol extraction. Revista Mexicana de Ingeniería Química 10(2): 301-312.

Serrato-Cruz, M.A. \& S. Miranda-Colin. 1998. Efectos de la domesticación en algunas características florales del cempoalxóchitl. Horticultura 4(1): 57-62.

Serrato-Cruz, M.A. \& J.S. Barajas-Pérez. 2006. Poblaciones silvestres de Tagetes filifolia Lag. en el centro y sur de México. Revista Fitotecnia Mexicana 29(2): 7-12.

Turner, B. L. 1996. Thecomps of México. Tageteae and Anthemideae. Phytologia Memoirs 6(10): 51-69.

Vasudevan P., S. KashyaP \& S. Sharma. 1997. Tagetes: a multipurpose plant. Centre for rural development and apropiate technology. Indian Institute of Technology 62(1-2): 29-35.

Villareal, J.A. \& J.L. Villaseñor. 2004. Flora de Veracruz: Compositae y tribu Tagetae. Instituto de Ecología y University of California 135: 1-67.

YANO T. 1996. Fractal Nature of Food Materials. Bioscience, Biotechecnology and Biochemistry 60(5): 739-744. 